\title{
Linfadenectomía retroperitoneal lumboaórtica laparoscópica por vía extraperitoneal en cáncer testicular no seminoma*
}

\author{
Dr. OCTAVIO A. CASTILLO C. ${ }^{1}$
}

1 Unidad de Urología y Centro de Cirugía Robótica, Clínica INDISA.

Facultad de Medicina, Universidad Andrés Bello.

Santiago, Chile.

\begin{abstract}
Extraperitoneal laparoscopic retroperitoneal lymph node dissection in non-seminomatous testicular cancer

Objective: The aim is to describe the technique of extraperitoneal laparoscopic access for retroperitoneal lymph node dissection in a series of patients with testis cancer stage A. Material and Methods: The extraperitoneal approach was performed in 5 patients with stage A testicular cancer. The technique includes the creation of a totally extraperitoneal space, full exposition of the retroperitoneum and classic retroperitoneal lymph node dissection. We analyzed demographic data, histology, access and surgical complications, estimated blood loss and follow up. Results: The average age was 29.4 years old (22-41). The mean operative time was 144 minutes, with an estimated blood loss of $42.4 \mathrm{ml}$. There were no surgical complications. The average hospital stay was $33.6 \mathrm{hr}$, and mean number of lymph nodes was 27.4 (24 -32). In long-term follow up there was no recurrence. Discussion: The extraperitoneal approach is an alternative access for retroperitoneal lymph node dissection in testis cancer patients. It allows avoiding potential intestinal lesions and there is no contraindication in patients with prior abdominal surgery.
\end{abstract}

Key words: Retroperitoneal lymph node dissection, testis cancer, laparoscopy, extraperitoneal.

\section{Resumen}

Objetivo: Mostrar la experiencia en la técnica de disección lumboaórtica por vía totalmente extraperitoneal, en un grupo de pacientes con cáncer testicular en estadio A. Material y Métodos: La serie está formada por 5 pacientes, portadores de un tumor testicular no seminoma, en estadio A. En ellos se planteó como alternativa la linfadenectomía retroperitoneal lumboaórtica laparoscópica. La técnica quirúrgica consistió en la formación de un espacio extraperitoneal, con rechazo de peritoneo, exposición del retroperitoneo y disección linfática clásica. Se analizaron los datos demográficos, histología, complicaciones del acceso y la

*Recibido el 25 de julio de 2012 y aceptado para publicación el 24 de septiembre de 2012.

$\mathrm{El}$ autor no refiere conflictos de interés.

Correspondencia: Dr. Octavio A. Castillo C.

Av. Santa María 1810, Santiago, Chile. 7520440

octavio.castillo@indisa.cl 
técnica quirúrgica, tiempo operatorio, sangrado estimado y seguimiento a largo plazo. Resultados: El tiempo operatorio medio fue de $144 \mathrm{~min}$, con un sangrado medio estimado en 42,5 $\mathrm{ml}(20-150 \mathrm{ml})$. No hubo complicaciones intra ni postoperatorias. El tiempo medio de hospitalización fue de 33,6 h. El número medio de nodos linfáticos resecados fue de 27,4 (24 a 32). Con un promedio de seguimiento de 134 meses, no ha habido recurrencia retroperitoneal ni diseminación a distancia. Discusión: La vía extraperitoneal es una alternativa de acceso para la disección linfática retroperitoneal en pacientes con cáncer testicular. Permite evitar potenciales lesiones intestinales y es factible de realizar en pacientes con cirugía abdominal previa.

Palabras clave: Cáncer testicular, linfadenectomía, lumboaórtica, laparoscopia, extraperitoneal.

\section{Introducción}

La linfadenectomía retroperitoneal lumboaórtica en cáncer de testículo ha superado la prueba del tiempo, siendo aún una de las alternativas de manejo de los tumores testiculares no seminoma ${ }^{1}$.

Con la identificación de factores de riesgo de enfermedad metastásica en la biopsia testicular (principalmente invasión vascular-linfática y predominancia de Carcinoma embrionario), el uso de marcadores (Alfafetoproteína, Subunidad Beta de Gonadotrofina coriónica y Láctico Deshidrogenasa), más los avances en imagenología (Tomografía axial computada, Resonancia magnética y Pet-Scan), han empezado a predominar los programas de vigilancia activa $^{2}$ y Quimioterapia primaria ${ }^{3}$ en base a 2 ciclos del esquema habitual de Bleomicina, Etopósido y CisPlatino (BEP). Además, una de las razones para evitar la disección retroperitoneal en paciente joven, es la morbilidad asociada a la cirugía y la eventual pérdida de la eyaculación.

El conocimiento anatómico de la inervación simpática y la distribución de las ramas responsables de la eyaculación, más la aparición de técnicas de mínima invasión, permitieron reducir significativamente la morbilidad quirúrgica y preservar la eyaculación ${ }^{4}$.

Nuestra experiencia con linfadenectomía retroperitoneal lumboaórtica laparoscópica por vía transperitoneal ha mostrado resultados satisfactorios, con baja morbilidad, preservación de la eyaculación y resultados oncológicos comparables con la cirugía abierta tradicional ${ }^{5-7}$.

Con el propósito de disminuir el riesgo de morbilidad, principalmente el riesgo de lesiones intestinales y de adherencias, es que aplicamos en un grupo de pacientes el acceso al retroperitoneo por vía totalmente extraperitoneal ${ }^{8,9}$.

\section{Material y Métodos}

La serie está constituida por 5 pacientes portadores de un tumor testicular no seminoma, en estadio clínico 1 . Todos ellos fueron operados por el mismo cirujano, luego de aprobación del Comité de Ética y aprobación del consentimiento informado.
Factores de riesgo presentes fueron Carcinoma embrionario predominante en 3 casos e invasión vascular-linfática en 1 .

Se analizaron los parámetros demográficos, complicaciones del acceso, dificultades técnicas intraoperatorias, complicaciones intra y post-operatorias, número de nodos linfáticos resecados y seguimiento a largo plazo.

\section{Técnica quirúrgica}

Ningún paciente tuvo preparación previa, excepto ayuno de $8 \mathrm{~h}$. Se colocó sonda nasogástrica y sonda vesical, las cuales se sacaron en la sala de recuperación postoperatoria.

El paciente se colocó en posición de flanco, con el lado del tumor original hacia arriba.

Se realiza una incisión transversal de $2 \mathrm{~cm}$ pararectal y para-umbilical (Figura 1), se abre la aponeurosis y se diseca el espacio pre-peritoneal en forma digital (Figura 2). Luego se introduce un balón de disección el cual se llena con 250 cc de solución fisiológica (Figura 3). Se coloca un trocar de Hasson y se introduce la óptica de $30^{\circ}$, completando la disección con movimientos de la óptica rechazando el peritoneo. Los trocares de trabajo se colocan bajo visión, generalmente uno en fosa ilíaca y uno en posición subcostal (Figura 4). Trocares adicionales pueden ir en el flanco o en el hipogastrio.

La disección retroperitoneal se realiza con la misma técnica descrita previamente para acceso transperitoneal ${ }^{7}$.

\section{Resultados}

La edad promedio de los pacientes fue de 29,4 años, con un rango de 22 a 41 años. El IMC promedio fue de 24,5 y todos los pacientes eran ASA 1. El lado del tumor fue izquierdo en 4 casos y derecho en 1 caso.

El tiempo operatorio medio fue de 144 min (105$240 \mathrm{~min}$ ), con un sangrado medio estimado en 42,5 $\mathrm{ml}(20-150 \mathrm{ml})$. No hubo complicaciones intra $\mathrm{ni}$ postoperatorias. El tiempo medio de hospitalización fue de 33,6 h (24-36 h).

El número medio de nodos linfáticos resecados fue de 27,4, con un rango de 24 a 32 nodos. 


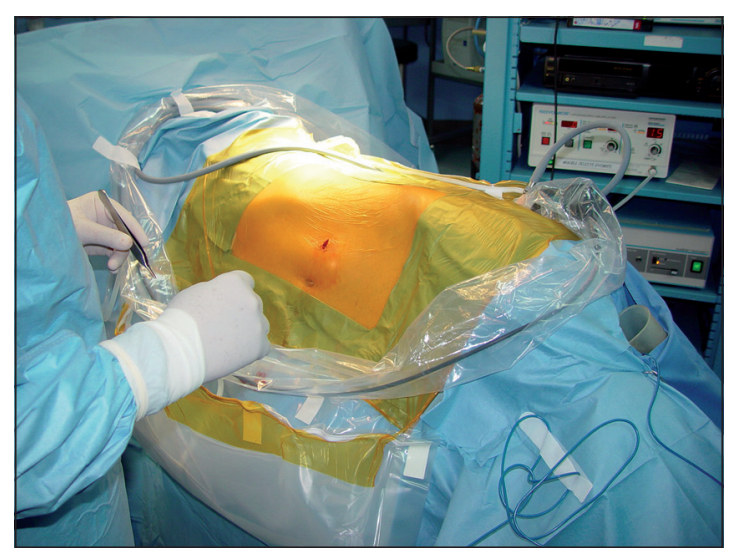

Figura 1. Incisión.

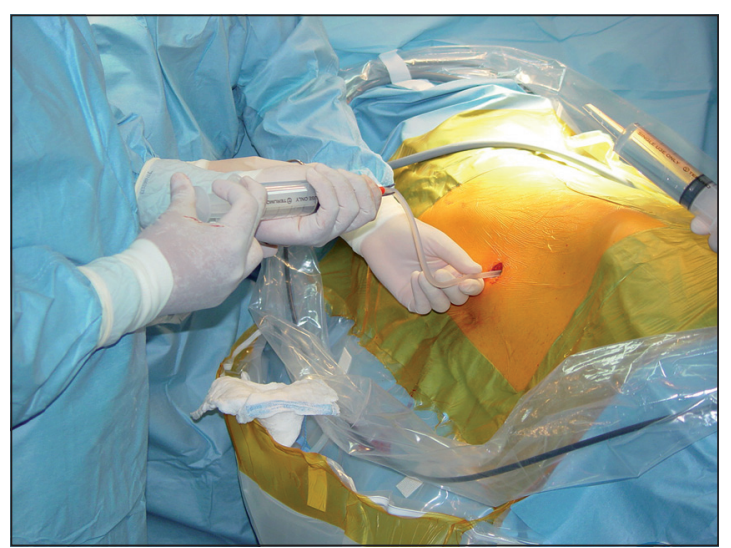

Figura 3. Disección con balón.

En un paciente se demostró metástasis de Carcinoma embrionario en 1 de 24 nodos linfáticos, realizando quimioterapia complementaria con 2 ciclos de BEP.

Con un promedio de seguimiento de 134 meses, no ha habido recurrencia retroperitoneal ni diseminación a distancia.

\section{Discusión}

La Linfadenectomía lumboaórtica en cáncer de testículo continúa siendo una excelente alternativa de manejo de los pacientes con cáncer testicular no seminoma en estadio 1, como método seguro de estadificación patológica. Tiene, además, un lugar fundamental, en el rescate de masas post-quimioterapia, y es de elección ante la presencia de tumores del tipo Teratoma, los cuales son quimio resistentes ${ }^{1,10}$.

La crítica más importante, es que se estaría haciendo una cirugía de alta morbilidad en pacientes

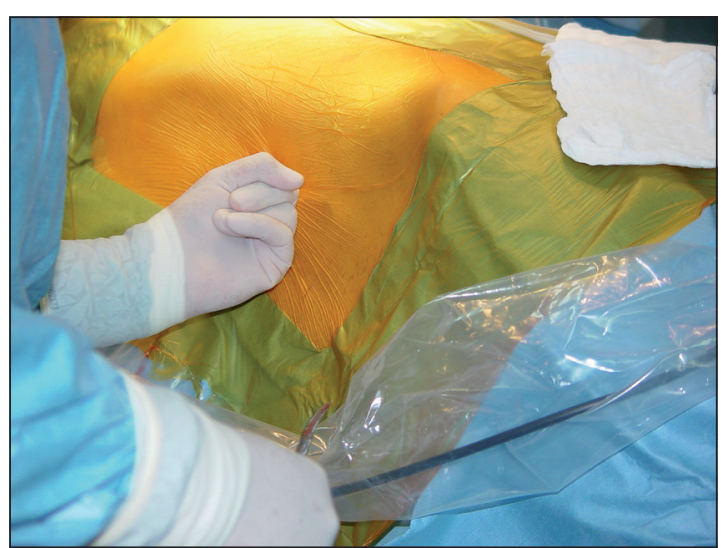

Figura 2. Disección digital.

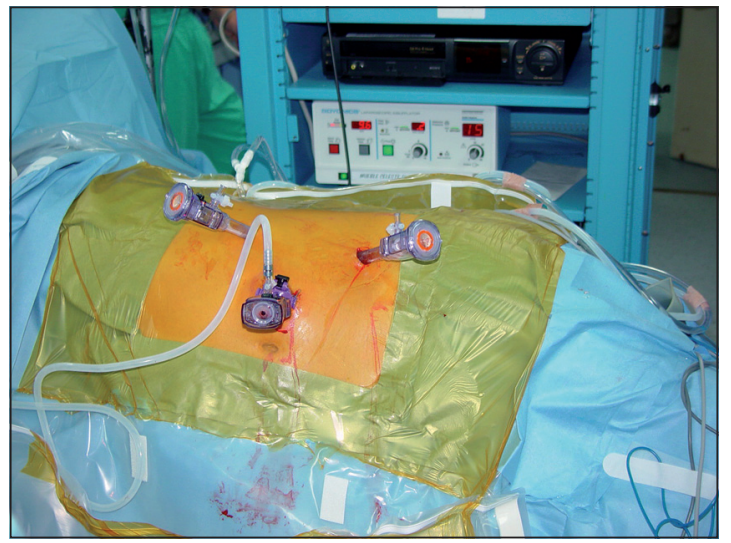

Figura 4. Trocares.

con riesgo de enfermedad metastásica retroperitoneal no superior al 30\%. Sin embargo, en estos pacientes el riesgo de recurrencia a distancia es de sólo $10 \%$ y el programa de seguimiento a largo plazo es más simple ${ }^{11}$. Mantiene no sólo un rol estadificador si no que también terapéutico, pudiendo evitarse el tratamiento quimioterápico en un grupo importante de pacientes. Los protocolos de vigilancia tiene una tasa de recurrencia de $30 \%$; en el $35 \%$ de los casos los marcadores tumorales son normales y aproximadamente el $60 \%$ de estas recurrencias ocurre en el retroperitoneo ${ }^{12-14}$. En los esquemas de quimioterapia primaria o ayudante, la recurrencia baja al 2,7\%, pero existe el problema de los efectos deletéreos a largo plazo (más de 20 años) como son la aparición de cánceres secundarios y la probabilidad alta de enfermedad cardiovascular y síndrome metabólico ${ }^{15-18}$.

Una de las críticas más importantes ha sido la utilización de una disección limitada, dado que las metástasis linfáticas retroperitoneales del cáncer testicular siguen un patrón definido, según el lado del 
tumor primario ${ }^{19-21}$. La utilización de estas plantillas de disección puede dejar con tratamiento incompleto a un grupo importante de pacientes con enfermedad por fuera de ellas ${ }^{22}$.

El conocimiento de la anatomía de las fibras simpáticas de la eyaculación, ha permitido hacer disecciones prolijas, evitando la ausencia de eyaculación en más del $90 \%$ de los casos. Sin embargo, la cirugía abierta tradicional sigue teniendo la morbilidad relacionada a una cirugía de alta complejidad, con grandes incisiones, movilización de colon y mesenterio del intestino delgado, larga exposición peritoneal con los consabidos problemas de íleo postoperatorio prolongado, dolor, larga hospitalización y plazos prolongados de reintegro al trabajo ${ }^{21}$.

Con el propósito de lograr mantener las ventajas de la cirugía laparoscópica pero realizando una Linfadenectomía retroperitoneal comparable a la cirugía abierta, en el año 1993 comenzamos una serie de pacientes operados por esa vía, resultados preliminares comunicados en el año $1994^{5}$.

Desde entonces nuestra experiencia se ha ido ampliando significativamente, habiendo demostrado que hemos podido imitar la técnica quirúrgica tradicional con el agregado de las ventajas ya conocidas de la cirugía laparoscópica ${ }^{6,7}$.

En nuestra experiencia hemos ido evolucionando con la técnica, disminuyendo las complicaciones y realizando ahora una disección bilateral, con el propósito potencial de lograr no sólo una adecuada estadificación, si no que también logar el efecto terapéutico deseado, evitando tratamientos complementarios.

En esta idea es que probamos realizar la disección retroperitoneal por una vía totalmente extraperitoneal. Fue primero descrita para biopsia linfática retroperitoneal en cáncer cérvico-uterino en el año $1994^{8}$, y para disección retroperitoneal para cáncer testicular en el año $2003^{9}$. Sus potenciales ventajas, al evitar la entrada a la cavidad peritoneal son disminución del riesgo de lesión intestinal, disminución de la incidencia de adherencias, disminución del íleo postoperatorio y la factibilidad de realizar en pacientes con cirugía abdominal previa.

Otra ventaja adicional es que esta técnica puede ser hecha en decúbito totalmente supino, evitando eventuales lesiones neurológicas debidas a inadecuada protección de las zonas de contacto en la posición de flanco utilizada habitualmente ${ }^{23}$.

Esta pequeña serie muestra la factibilidad de realizar una adecuada disección retroperitoneal oncológica por vía totalmente extraperitoneal. La curva de aprendizaje es corta y el resto de la técnica es igual que por la vía transperitoneal, pero evitando la movilización del intestino.

La introducción de la cirugía robótica nos ha llevado a realizar las primeras experiencias de linfadenectomía retroperitoneal lumboaórtica con esta asistencia quirúrgica ${ }^{24}$. La versatilidad de este, permitirá con mayor facilidad aplicar la vía extraperitoneal para acceder al retroperitoneo en forma directa $^{25}$.

\section{Referencias}

1. Spermon JR, Roeleveld TA, van der Poel HG, Hulsbergen-van de Kaa CA, Ten Bokkel Huinink WW, van de Vijver M, et al. Comparison of surveillance and retroperitoneal lymph node dissection in stage I nonseminomatous germ cell tumors. Urology 2002;59:923-9.

2. Read G, Stenning SP, Cullen MH, Parkinson MC, Horwich A, Kaye SB, et al. Medical Research Council prospective study of surveillance for stage I testicular teratoma. J Clin Oncol. 1992;10:1762-8.

3. Cullen MH, Stenning SP, Parkinson MC, Fossa SD, Kaye SB, Horwich AH, et al. Short-course adjuvant chemotherapy in high-risk stage I nonseminomatous germ cell tumors of the testis: a Medical Research Council report. J Clin Oncol. 1996;14:1106-13.

4. Heidenreich A, Albers P, Hartmann M, Kliesch S, Kohrmann KU, Krege S, et al. Complications of primary nerve sparing retroperitoneal lymph node dissection for clinical stage I nonseminomatous germ cell tumours of the testis: experience of the German Testicular Cancer Study Group. J Urol. 2003;169:1710-4.

5. Castillo O, Wöhler C, Mayanz M, Van Cauwelaert R, Muñoz V, Aguirre C, et al. Laparoscopic lumboaortic retroperitoneal lymphadenectomy in testicular neoplasm]. Rev Med Chile 1994;122:313-8.

6. Castillo O, Rioja J, Vidal-Mora I, Feria M, SánchezSalas R. Morbilidad de la linfadenectomía lumboaórtica laparoscópica en pacientes con tumor testicular no seminomatoso en estadio clínico A. Rev Chil Cir. 2009;61:533-7.

7. Castillo OA, Sánchez-Salas R, Secin FP, Campero JM, Foneron A, Vidal-Mora I. Primary laparoscopic retroperitoneal lymph node dissection for clinical stage I nonseminomatous germ-cell testis tumor. Actas Urol Esp. 2011;35:22-8.

8. Vasilev SA, McGonigle KF. Extraperitoneal laparoscopic para-aortic lymph node dissection. Gynecol Oncol. 1996;61:315-20.

9. Hsu TH, Su LM, Ong A. Anterior extraperitoneal approach to laparoscopic retroperitoneal lymph node dissection: a novel technique J Urol. 2003;169:258-60.

10. Hendry WF, Norman A, Nicholls J, Dearnaley DP, Peckham MJ, Horwich A. Abdominal relapse in Stage 1 nonseminomatous germ cell tumours of the testis managed by surveillance or with adjuvant chemotherapy. BJU Int. 2000;86:89-93.

11. Freedman LS, Parkinson MC, Jones WG, Oliver RT, 
Peckham MJ, Read G, et al. Histopathology in the prediction of relapse of patients with stage I testicular teratoma treated by orchidectomy alone. Lancet 1987;2:294-8.

12. Colls BM, Harvey VJ, Skelton L, Frampton CM, Thompson PI, Bennett M, et al. Late results of surveillance of clinical stage I nonseminoma germ cell testicular tumours: 17 years' experience in a national study in New Zealand. Br J Urol Int. 1999;83:76-82.

13. Oliver RT, Ong J, Shamash J, Ravi R, Nagund V, Harper $\mathrm{P}$, et al. Long-term follow-up of Anglian Germ Cell Cancer Group surveillance versus patients with Stage 1 nonseminoma treated with adjuvant chemotherapy. Urology 2004;63:556-61.

14. Zúñiga A, Kakiashvili D, Jewett MA. Surveillance in stage I nonseminomatous germ cell tumours of the testis. BJU Int. 2009;104(9 Pt B):1351-6.

15. Oliver RT, Raja MA, Ong J, Gallagher CJ. Pilot study to evaluate impact of a policy of adjuvant chemotherapy for high risk stage I malignant teratoma on overall relapse rate of stage I cancer patients. J Urol. 1992;148:1453-6.

16. Studer UE, Fey MF, Calderoni A, Kraft R, Mazzucchelli L, Sonntag RW. Adjuvant chemotherapy after orchidectomy in high-risk patients with clinical stage I nonseminomatous testicular cancer. Eur Urol. 1993;23:444-9.

17. Böhlen D, Borner M, Sonntag RW, Fey MF, Studer UE. Long-term results following adjuvant chemotherapy in patients with clinical stage I testicular nonseminomatous malignant germ cell tumors with high risk factors. J Urol. 1999; 161:1148-52.

18. Chevreau C, Mazerolles C, Soulié M, Gaspard MH, Mourey L, Bujan L, et al. Long-term efficacy of two cycles of BEP regimen in high risk stage I nonsemi- nomatous testicular germ cell tumors with embryonal carcinoma and/or vascular invasion. Eur Urol. 2004;46:209-14.

19. Pizzocaro G, Salvioni R, Zanoni F. Unilateral lymphadenectomy in intraoperative stage I nonseminomatous germinal testis cancer. J Urol. 1985;134:485-9.

20. Donohue JP, Thornhill JA, Foster RS, Rowland RG, Bihrle R. Retroperitoneal lymphadenectomy for clinical stage A testis cancer (1965 to 1989): modifications of technique and impact on ejaculation. J Urol. 1993;149:237-43.

21. Weissbach L, Boedefeld EA. Localization of solitary and multiple metastases in stage II nonseminomatous testis tumor as basis for a modified staging lymph node dissection in stage I. J Urol. 1987;138:77-82.

22. Eggener SE, Carver BS, Sharp DS, Motzer RJ, Bosl GJ, Sheinfeld J. Incidence of disease outside modified retroperitoneal lymph node dissection templates in clinical stage I or IIA nonseminomatous germ cell testicular cancer. J Urol. 2007;177:937-42.

23. Hara I, Kawabata G, Yamada K, Tanaka K, Kamidono S. Extraperitoneal laparoscopic retroperitoneal lymph node dissection in supine position after chemotherapy for advanced testicular carcinoma. Int $\mathrm{J}$ Urol. 2004;11:934-9.

24. Castillo OA, Sepúlveda F, Susanibar LF, Vidal-Mora I, Rubio G. Linfadenectomía lumboaórtica asistida por robot en tumor residual post-quimioterapia en cáncer testicular. Rev Chil Cir. 2011;63:508-12.

25. Narducci F, Lambaudie E, Houvenaeghel G, Collinet $P$, Leblanc E. Early experience of robotic-assisted laparoscopy for extraperitoneal para-aortic lymphadenectomy up to the left renal vein. Gynecol Oncol. 2009;115:1724. 